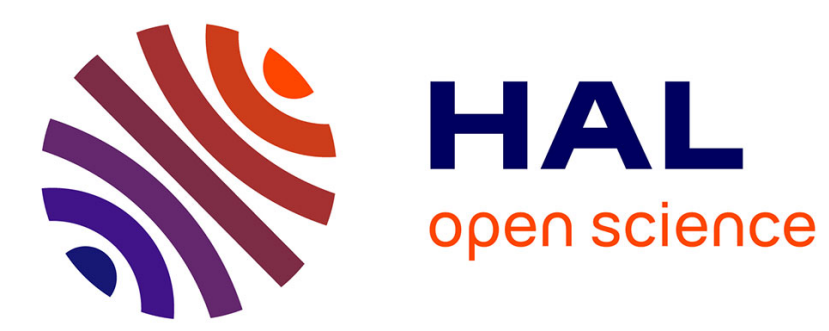

\title{
SIMULATIONS OF TWO-PHASE FLOWS WITH A MULTIFIELD APPROACH
}

Solène Fleau, Stéphane Mimouni, Nicolas Mérigoux, Stéphane Vincent

\section{To cite this version:}

Solène Fleau, Stéphane Mimouni, Nicolas Mérigoux, Stéphane Vincent. SIMULATIONS OF TWOPHASE FLOWS WITH A MULTIFIELD APPROACH. CHT-15, May 2015, New Brunswick, France. hal-01172289

\section{HAL Id: hal-01172289 \\ https://hal.science/hal-01172289}

Submitted on 9 Jul 2015

HAL is a multi-disciplinary open access archive for the deposit and dissemination of scientific research documents, whether they are published or not. The documents may come from teaching and research institutions in France or abroad, or from public or private research centers.
L'archive ouverte pluridisciplinaire HAL, est destinée au dépôt et à la diffusion de documents scientifiques de niveau recherche, publiés ou non, émanant des établissements d'enseignement et de recherche français ou étrangers, des laboratoires publics ou privés. 


\title{
SIMULATIONS OF TWO-PHASE FLOWS WITH A MULTIFIELD APPROACH
}

\author{
Solène Fleau ${ }^{*} \S$, Stéphane Mimouni ${ }^{*}$, Nicolas Mérigoux ${ }^{*}$, Stéphane Vincent ${ }^{*}$ \\ "Electricité de France, R\&D Division, Chatou, France. \\ *** Laboratoire de Modélisations et Simulations Multi-Echelle, Université Paris-Est, France. \\ ${ }^{\S}$ Correspondence author. Email: solene.fleau@edf.fr
}

\begin{abstract}
Safety issues in nuclear power plant involve complex bubbly flows. To predict the behavior of these flows, the two-fluid approach is often used. Nevertheless, this model induces a natural diffusion of interfaces, which results in a poor accuracy in the calculation of the local parameters. Therefore, to simulate large interfaces such as slugs or free surfaces, located methods have been developed using the single-fluid model. In this paper, the two approaches have been coupled in the CMFD code NEPTUNE_CFD to simulate adiabatic separated flows. The averaged momentum balance equations are solved for each field and are followed by an artificial compression step, which fixes the interface thickness and ensures mass conservation. Moreover, since the two-fluid model allows the existence of relative velocities at the interface, a drag force is used to cancel them. This article proposes also a new formulation for this force, to take into account the physical properties of the flow. To validate this approach, an analytical test case with a static bubble has been simulated with a mesh refinement test. Then, the simulation of the Kelvin-Helmholtz instability has been performed to highlight the effect of the modification of the drag force. The sheared interface is particularly sensible to this force, which has an important influence on the flow parameters such as the interface velocity. Finally, these developments have been compared to three other codes by simulating the RayleighTaylor instability.
\end{abstract}

\section{NOMENCLATURE}

\begin{tabular}{|lll|}
\hline \multicolumn{2}{|l|}{ Roman letters: } \\
\hline $\mathrm{C}$ & - & Circularity \\
$C_{d}$ & - & Drag coefficient \\
$d_{p}$ & $\mathrm{~m}$ & Characteristic length scale \\
$d_{p d i s p}$ & $\mathrm{~m}$ & Diameter of the dispersed bubbles/droplets \\
$\mathrm{I}$ & $\mathrm{kg} \cdot \mathrm{m}^{-2} \cdot \mathrm{s}^{-2}$ & Interfacial momentum transfer \\
$\mathrm{k}$ & $\mathrm{m}^{-1}$ & Wavenumber \\
$\mathrm{L}$ & $\mathrm{m}$ & Perimeter \\
$\mathbf{n}$ & - & Unit interface normal vector \\
$\mathrm{T}$ & $\mathrm{Pa}$ & Viscous stress tensor \\
$\mathbf{U}$ & $\mathrm{m} / \mathrm{s}$ & Average interface velocity \\
\hline
\end{tabular}




\begin{tabular}{|lll|}
\hline \multicolumn{2}{|l|}{ Greek letters: } \\
\hline$\Delta x$ & $\mathrm{~m}$ & $\begin{array}{l}\text { Cube root of the cell } \\
\text { volume }\end{array}$ \\
$\kappa$ & $\mathrm{m}^{-1}$ & Interface curvature \\
$\Gamma$ & $\mathrm{W} / \mathrm{m}^{3}$ & Interfacial mass transfer \\
$\omega$ & $\mathrm{s}^{-1}$ & Pulsation \\
$\sigma$ & $\mathrm{N} / \mathrm{m}$ & Surface tension coefficient \\
$\Omega$ & $\mathrm{m}^{3}$ & Volume element \\
$\tau$ & $\mathrm{s}$ & Characteristic time scale \\
\hline
\end{tabular}

\begin{tabular}{|ll|}
\hline \multicolumn{2}{|l|}{ Subscripts and superscripts: } \\
\hline int & Interface \\
$\mathrm{i}$ & Space direction \\
$\mathrm{j}$ & Cell index \\
$\mathrm{k}$ & Phase index \\
\hline
\end{tabular}

\section{INTRODUCTION}

Many situations in nuclear power plant are characterized by liquid vapor interfaces. Whereas these flows are well controlled at normal conditions, they could threaten the integrity of the reactor pressure vessel and conduct to a contamination of the environment with radioactive nuclei in case of hypothetical accidents. Therefore, important investigations are carried out to understand these complex flows.

Bubbly flows occurring in nuclear power plant are often modelled with an Eulerian dispersed description within the two-fluid model of Ishii [1975]. In this method, the bubbles are small enough to be considered spherical. Thus, interfacial forces such as drag force, lift force, wall lubrication, virtual mass and turbulent dispersion force are implemented between the continuous field and these dispersed bubbles [e.g., Mimouni et al. 2011]. This approach seems then inappropriate to simulate larger and deformable bubbles. In fact, the interface location cannot be accurate due to the numerical diffusion.

Therefore, large interfaces are usually simulated through located approaches using single-fluid models such as front tracking [e.g., Unverdi and Tryggvason 1992], level-set [e.g., Sussman et al. 1994] or Volume Of Fluid (VOF) [e.g., Hirt and Nichols 1981]. The VOF approach has been extensively used for the simulation of two-phase flows because mass conservation is ensured. Nevertheless, its low order of accuracy conducted the researchers to find alternatives. Thus, Unverdi and Tryggvason [1992] demonstrated the usefulness of the front tracking method on film boiling simulations. But, the algorithm has been criticized for its difficulty to follow topology changes at vapor-liquid interfaces. More recently, the level set methods have become interesting for their high order of accuracy. Nevertheless, research has been necessary to deal with their problem of mass conservation, highlighted by Enright et al. [2002]. Thus, Olsson and Kreiss [2005] proposed a conservative scheme to solve the advection equation of the level set function.

In recent works, these two approaches have been combined to take advantages of both of them. The fluid motion is simulated within the two-fluid model whereas interfaces are located with a conservative level set method. The balance equations are solved for each field using the interfacial properties, accurately evaluated thanks to the level set method. Thus, Štrubelj [2009] implemented this method in his in-house code to simulate different stratified flows. His comparison with other codes highlighted the effect of the method on the interface smearing. Lately, Zuzio et al. [2013] applied the method to a primary atomization process.

This method have become crucial over the last decades with the development of a new approach to simulate more accurately complex flows, which can contain at the same time bubbles with a large 
range of sizes and free surfaces. This approach, called the multifield approach, is based on the concept of a four field and a two-fluid model [e.g., Lahey and Drew 2001]. In this context, the accurate location of the large interfaces is crucial since the flow properties are highly influenced by these structures.

In this paper, we adapt the above method in the CMFD code NEPTUNE_CFD [e.g., Mimouni et al. 2014]. For this purpose, the interface sharpening equation developed by Olsson and Kreiss [2005] is implemented. Furthermore, a new drag force expression is proposed to take more into account the flow properties such as the fluid viscosity.

In this article, we will first describe the equations of the two-fluid model in the case of two separated phases. The different relevant forces necessary for the simulation of this kind of flow will also be exposed. To validate this approach, a static bubble will be simulated with different mesh refinements. Then, a simulation of the Kelvin-Helmholtz instability in the Thorpe's experiment configuration [e.g., Thorpe 1969] will be performed to highlight the effect of the drag force. Finally, these new developments implemented in the code NEPTUNE_CFD will be compared to other codes with the simulation of the Rayleigh-Taylor instability.

\section{COMPUTATIONAL MODEL}

Two-fluid model The code NEPTUNE_CFD is based on an Eulerian approach with a finite volume discretization. The flow motion is followed using the two-fluid model of Ishii [1975] extended to nphase. In this model, the density, the viscosity and the local velocity are defined for each field in each cell j.

This study is restricted to incompressible, laminar and adiabatic cases. Thus, no thermal energy is exchanged and no turbulence models are used. The following governing equations are solved for each phase k:

- The mass balance equation :

$$
\partial_{t} \varepsilon_{k}^{*} \rho_{k}+\partial_{x_{i}}\left(\varepsilon_{k}^{*} \rho_{k} u_{i, k}\right)=\Gamma_{k}
$$

- The momentum equation in each space direction $\mathrm{i}$ :

$$
\begin{aligned}
\partial_{t}\left(\varepsilon_{k}^{*} \rho_{k} u_{i, k}\right)+\partial_{x_{j}}\left(\varepsilon_{k}^{*} \rho_{k} u_{i, k} u_{j, k}\right)= & \partial_{x_{j}}\left(\varepsilon_{k}^{*} T_{i j, k}\right)-\varepsilon_{k}^{*} \partial_{x_{j}} P \\
& +\varepsilon_{k}^{*} \rho_{k} g_{i}+I_{i, k}+F_{i, k}
\end{aligned}
$$

where $F_{i, k}$ denotes the extra source terms such as surface tension or drag models, which will be introduced further.

Conservation of volume, mass and momentum leads to three others equations:

$$
\begin{gathered}
\sum_{k} \varepsilon_{k}^{*}=1 \\
\sum_{k} \Gamma_{k}=0 \\
\sum_{k} I_{k}=\frac{1}{\Omega} \int_{i n t} \sigma \kappa^{i n t} n^{i n t} d S
\end{gathered}
$$


These equations do not allow to close the system. Therefore, in the code NEPTUNE_CFD, the assumption of a common pressure for all fields is made:

$$
\forall \mathrm{k}, \mathrm{P}_{\mathrm{k}}=\mathrm{P}
$$

Finally, models are used for the interfacial transfers.

Surface tension Clift et al. [1978] highlighted the necessity of surface tension forces to deal with flows containing large interfaces. Because the interface has a finite thickness in our approach, the choice is made to use the Continuum Surface Force (CSF) model proposed by Brackbill et al. [1992]:

$$
\boldsymbol{F}_{\boldsymbol{C S F}}=\sigma \kappa \mathbf{n}
$$

with $\mathbf{n}$ the interface normal vector:

$$
\boldsymbol{n}=\frac{\nabla \varepsilon_{k}^{*}}{\left\|\nabla \varepsilon_{k}^{*}\right\|}
$$

and $\kappa$ the local curvature:

$$
\kappa=-\nabla \cdot\left(\frac{\nabla \varepsilon_{k}^{*}}{|| \nabla \varepsilon_{k}^{*}||}\right)
$$

However, since the code NEPTUNE_CFD is based on the two-fluid model, the Continuum Surface Force has to be splitted between the two fields. Therefore, the expression of the volumetric force becomes [e.g., Bartosiewicz et al. 2008]:

$$
\boldsymbol{F}_{\boldsymbol{C S} \boldsymbol{F}}=\beta_{\mathrm{k}} \sigma \kappa \nabla \varepsilon_{k}^{*} \mathrm{dV}
$$

The coefficient $\beta_{\mathrm{k}}$ is chosen equal to $\varepsilon_{k}^{*}$. In fact, Štrubelj [2009] compared this formulation to a mass formulation by simulating a pressure jump over a droplet interface. In this test case, the density ratio is large and surface tension plays a dominant role. He showed that the differences between the two models were minimal but the volume averaging still gave better results.

Drag force In the two-fluid model, the drag force is crucial. Contrary to the single fluid approach, with this model, two different velocities are defined, one for each phase. Therefore, at the interface, these two velocities can have two different values. The role of this force is then to ensure the equality of these velocities at the interface. The usual expression is [e.g., Strubelj 2009]:

$$
\mathbf{F}_{\text {Drag }}=\varepsilon_{l}^{*} \varepsilon_{g}^{*} \frac{\varepsilon_{l}^{*} \rho_{l}+\varepsilon_{g}^{*} \rho_{g}}{\tau}\left(\boldsymbol{u}_{\boldsymbol{l}}-\boldsymbol{u}_{\boldsymbol{g}}\right)
$$

In a first approach, $\tau$ was taken equal to $1.10^{-7} \mathrm{~s}$. Nevertheless, Denèfle [2014] simulated the experiment of Raymond and Rosant [2000] with this drag force. He showed that the results were in better agreement with the experimental data for cases with higher viscosities. 
Therefore, we developed a new expression. The new formulation is based on Equation (11) and used the Ishii's definition of $\tau$ [e.g., Ishii 1975]:

$$
\frac{1}{\tau}=\frac{3 \rho_{l} C_{d}}{4 \rho_{g} d_{p}}\left\|\boldsymbol{u}_{\boldsymbol{l}}-\boldsymbol{u}_{\boldsymbol{g}}\right\|
$$

The drag coefficient $C_{d}$ is then defined by the Schiller and Nauman equation [e.g., Schiller and Nauman 1935]. Finally, the following drag force expression is obtained:

$$
\begin{array}{cc}
\varepsilon_{g}^{*}<0.3: & \mathbf{F}_{\text {bubble }}=\varepsilon_{l}^{*} \varepsilon_{g}^{*} \frac{\mu_{l}}{\mu_{g}} \frac{18 \mu_{l}}{\varepsilon_{l}^{*} d_{p}^{2}}\left(\boldsymbol{u}_{\boldsymbol{l}}-\boldsymbol{u}_{\boldsymbol{g}}\right) \\
\varepsilon_{g}^{*}>0.7: & \mathbf{F}_{\text {droplet }}=\varepsilon_{l}^{*} \varepsilon_{g}^{*} \frac{\mu_{l}}{\mu_{g}} \frac{18 \mu_{g}}{\varepsilon_{l}^{*} d_{p}^{2}}\left(\boldsymbol{u}_{\boldsymbol{l}}-\boldsymbol{u}_{\boldsymbol{g}}\right) \\
0.3 \leq \varepsilon_{g}^{*} \leq 0.7: & \mathbf{F}_{\text {mix }}=\frac{0.7-\varepsilon_{g}^{*}}{0.7-0.3} \mathbf{F}_{\text {bubble }}+\frac{\varepsilon_{g}^{*}-0.3}{0.7-0.3} \mathbf{F}_{\text {droplet }}
\end{array}
$$

The peculiarity of this new definition is that the viscosity becomes a parameter of the drag force intensity. Moreover, the new drag force anticipates the concept of the multifield approach, recently developed for the simulation of complex flows [e.g., Lahey and Drew 2001]. In fact, the expression is adapted to deal with a continuous and a dispersed field for each phase, in the same flow with possible mass exchanges between them. Smooth transitions between these fields are ensured by the definition of $\mathrm{F}_{\mathrm{mix}}$.

The multifield model is also taken into account in the definition of the characteristic distance $d_{p}$. The large interfaces and the small spherical bubbles, which belongs to a dispersed field are distinguished according to the value of $\varepsilon_{l}^{*} \varepsilon_{g}^{*}$. Thus, in the region of large interfaces, $\varepsilon_{l}^{*} \sim \varepsilon_{g}^{*} \sim 0.5$, which means that $\varepsilon_{l}^{*} \varepsilon_{g}^{*} \sim 0.25$. In this domain, $d_{p}$ is taken equal to $\frac{\varepsilon_{k}^{*}}{\left\|\nabla \varepsilon_{k}^{*}\right\|}$. On the contrary, in cells with dispersed gas for example, $\varepsilon_{g}^{*}<\varepsilon_{l}^{*}$ with $\varepsilon_{l}^{*}>0.95$. Therefore, for $\varepsilon_{l}^{*} \varepsilon_{g}^{*}<0.05, d_{p}$ is chosen equal to the diameter of the dispersed bubbles/droplets. Between these two regions, $d_{p}$ is evaluated by interpolation of its two extreme values :

$$
d_{p}=\frac{\varepsilon_{l}^{*} \varepsilon_{g}^{*}-b}{a-b} d_{p d i s p}+\frac{\varepsilon_{l}^{*} \varepsilon_{g}^{*}-a}{b-a} \frac{\varepsilon_{k}^{*}}{\left\|\nabla \varepsilon_{k}^{*}\right\|}
$$

with $\mathrm{a}=0.05$ and $\mathrm{b}=0.25$. An illustration of the method chosen to define $d_{p}$ is proposed in Figure 1 . This distribution of the characteristic distance $d_{p}$ ensures a smooth transition between this drag force, applied to the interfaces separated two continuous fields, and the drag force between a dispersed and a continuous field.

Finally, in the new drag force expression, we can notice the adding factor $\frac{\mu_{l}}{\mu_{g}}$, which is not obtained theoretically. In fact, simulation results showed that this factor was necessary to reach an intensity of the drag force, which is able to cancel the velocity differences between the two continuous fields at the interface. 


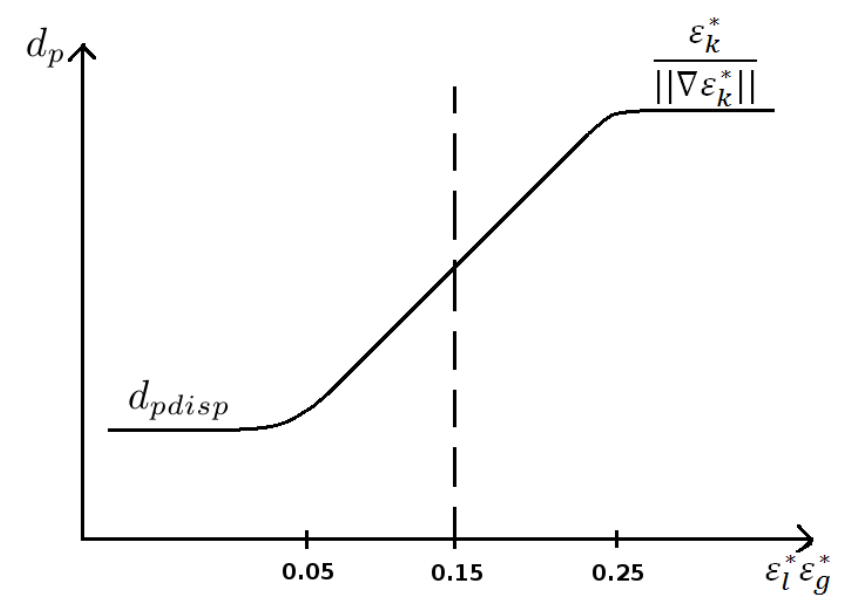

Figure 1. Repartition of $d_{p}$ along the domain, large interfaces are located at high values of $\varepsilon_{l}^{*} \varepsilon_{g}^{*}$.

Interface sharpening In the code NEPTUNE_CFD, the interface is located using a color function. Nevertheless, in the two-fluid model, the natural diffusion of this interface does not allow an accurate calculation of the interface properties such as the local curvature and the interface normal vector. Therefore, we locally solve the artificial compression equation proposed by Olsson and Kreiss [2005]:

$$
\partial_{\tau} \varepsilon_{k}^{*}+\nabla\left(\varepsilon_{k}^{*}\left(1-\varepsilon_{k}^{*}\right) n\right)=\epsilon \Delta \varepsilon_{k}^{*}
$$

The viscosity term $\epsilon \Delta \varepsilon_{k}^{*}$ was added by Olsson and Kreiss [2005] to prevent discontinuities at the interface. The value of the parameters $\Delta \tau$ and $\epsilon$ is chosen to obtain a final interface width always equal to 5 cells whatever the initial interface diffusion, as shown in Figure 2. Strubelj [2009] proposed:

$$
\Delta \tau=\frac{\Delta x}{32} \text { and } \epsilon=\frac{\Delta x}{2}
$$

Numerical scheme A Semi-Implicit Method for Pressure-Linked Equations (SIMPLE) solver is used in the code NEPTUNE_CFD [e.g., Patankar and Spalding 1972]. This algorithm can be summarized as followed:

- Set the boundary conditions,

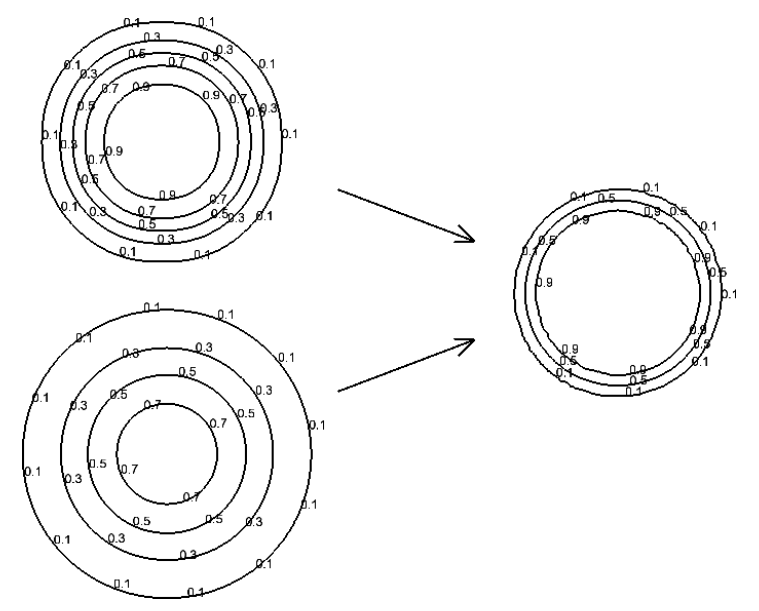

Figure 2. Sensitivity to the sharpening method. 
- Evaluate the gradients of velocity and pressure,

- Solve the discretized momentum equation to estimate an intermediate velocity field,

- Compute the intermediate mass fluxes at cell faces,

- Solve the pressure correction equation,

- Update the pressure field by taking into account the pressure correction,

- Solve the interface sharpening equation,

- Correct the face mass fluxes,

- Correct the velocity field using the pressure correction,

- Update the densities.

An iterative coupling of the equations is applied to ensure both mass and energy conservation. The data structure is face-based to allow simulations on arbitrary-shaped cells including nonconforming meshes.

\section{SIMULATION RESULTS}

In this part, we will validate the approach developed above on three different test cases. Beginning with a validation test case including a mesh refinement analysis, we will then compare the effect of the modification of the drag force expression with the simulation of the Thorpe's experiment [e.g., Thorpe 1969]. Finally, the results of the simulation of the Rayleigh-Taylor instability will be compared with other codes.

Static bubble For this test case, an air bubble is simulated in still water with an initial round shape and without gravity forces. Its initial diameter is taken equal to $2 \mathrm{~cm}$. The mesh is a two-dimensional cube, with 5-cm sides. Six different mesh refinements are tested: 45 x 45 cells, 64 x 64 cells, 91 x 91 cells, $128 \times 128$ cells, $181 \times 181$ cells and $256 \times 256$ cells. The time step is constant and equal to $0.1 \mathrm{~ms}$ except for the more refined mesh, where the time step is equal to $0.05 \mathrm{~ms}$. Therefore, the CFL number is kept under 0.9. The simulations are performed for $0.1 \mathrm{~s}$. At this time, we assume that the bubble has reached its final radius and pressure.

The quantities observed are the relative error for the circularity $\mathrm{C}$, evaluated using Equation (17), for the pressure, defined by the Laplace in Equation (18), and the average bubble velocity.

$$
C=\frac{2 \pi R_{0}}{L}
$$

where $R_{0}$ is the final bubble radius equal to $2 \mathrm{~cm}$ and $\mathrm{L}$ the bubble perimeter at the end of the simulation.

$$
p_{\text {in }}-p_{\text {out }}=\frac{\sigma}{R_{0}}
$$

where $p_{\text {in }}$ is the pressure in the bubble and $p_{\text {out }}$ out of the bubble, $\sigma$ corresponds to the surface tension coefficient equal to $0.08 \mathrm{~N} / \mathrm{m}$.

All these parameters are plotted in Figure 3. The $\mathrm{X}$ axis of the three graphs corresponds to the dimensionless quantity obtained by dividing the bubble diameter by the cell length.

In this figure, we note a convergence at second order for the circularity and at first order for the average velocity. Therefore, the intensity of spurious velocities decreases with the mesh refinement and remains low even with coarse meshes. Concerning the pressure, it seems that no convergence is obtained. 

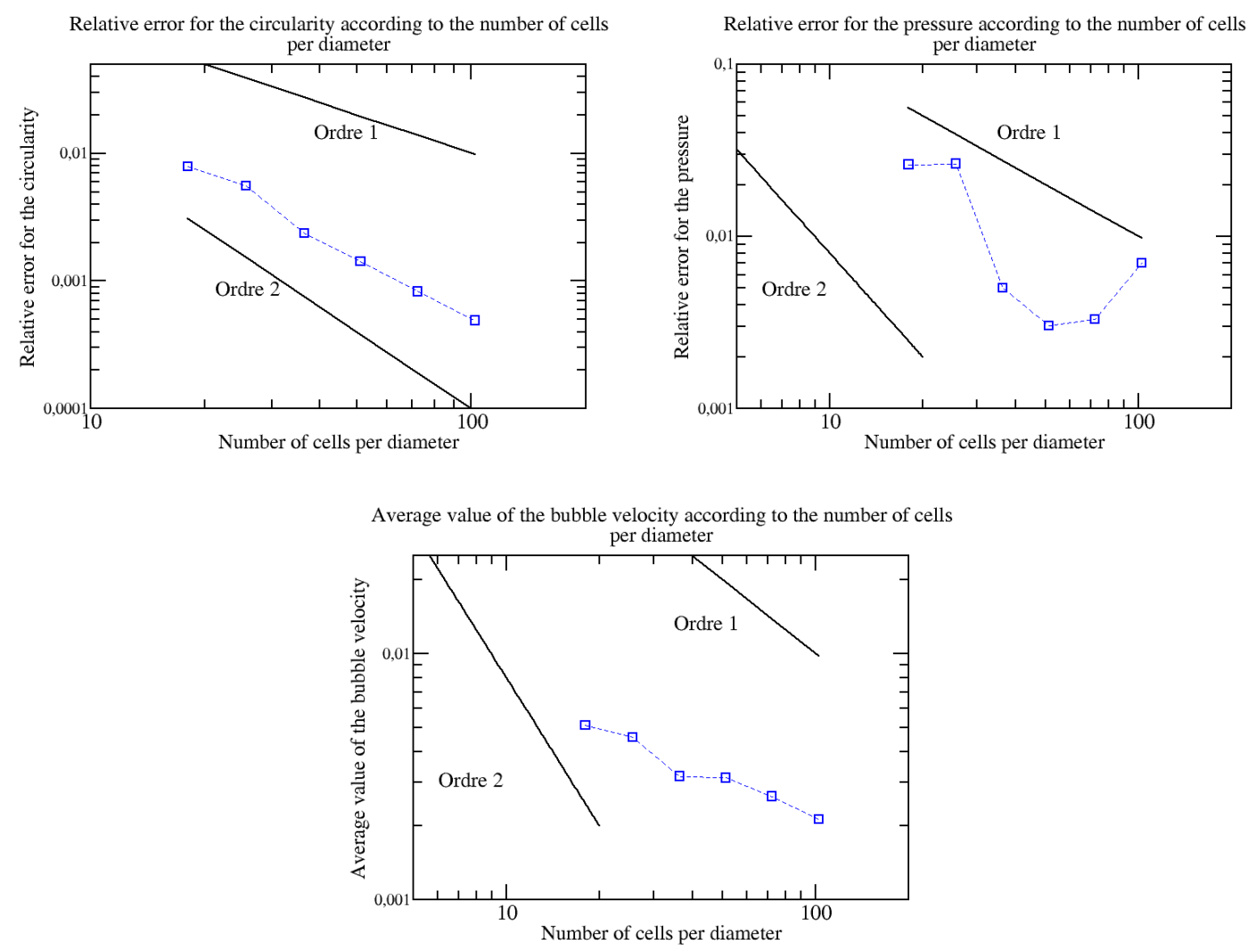

Figure 3. Effect of the mesh refinement on the relative error for the circularity and for the pressure and the average bubble velocity.

Kelvin-Helmholtz instability In this study, the Kelvin-Helmholtz instability is observed in the Thorpe's experiment configuration [e.g., Thorpe 1969]. Two immiscible fluids are contained in a rectangular box, which is tilted for a small angle, $\sin (\gamma)=0.072$, as displayed in Figure 4 . The KelvinHelmholtz instability is observed when the relative velocity between the two fluids exceeds a critical velocity.

In this test case, the velocity difference between the two fluids can be high in the bulk. Therefore, the two-fluid model can predict high relative velocities at the sheared interface. Thus, in this context, the drag force plays a crucial role. In fact, if the intensity of the drag force is inadequate, the simulated flow behavior can be dramatically affected.

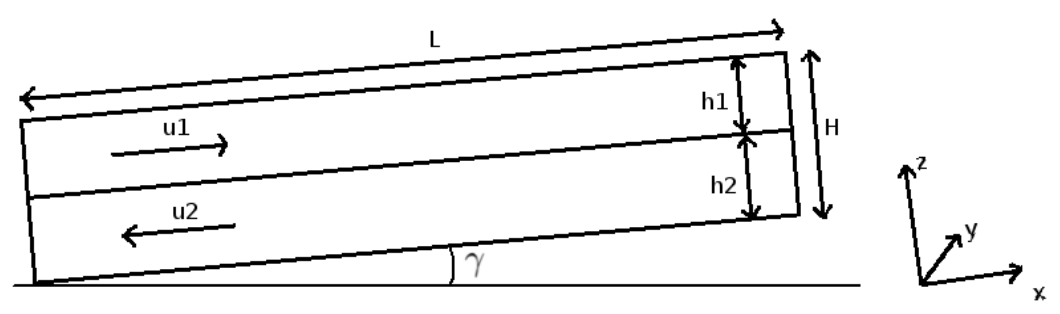

Figure 4. Schematic view of the Thorpe experiment at initial conditions [e.g., Thorpe 1969]. 
Theory. We consider here an inviscid fluid flow with:

$$
\rho= \begin{cases}\rho_{2} & \text { for } 0<z<h \\ \rho_{1} & \text { for } h<z<H\end{cases}
$$

with $h=h_{1}=h_{2}=\frac{H}{2}$.

The steady velocity distribution along the rectangular tube is:

$$
u=\left\{\begin{array}{l}
-\frac{\Delta u}{2} \text { for } 0<z<h \\
\frac{\Delta u}{2} \quad \text { for } h<z<H
\end{array}\right.
$$

This parallel flow is assumed to be a solution of Euler equations upon which is superposed a small perturbation proportional to $\exp (i(k x+\omega t))$. The linearization of the Euler equations gives the following dispersion relation:

$$
\omega=k \frac{\Delta u\left(\rho_{2}-\rho_{1}\right)}{2\left(\rho_{1}+\rho_{2}\right)} \pm \sqrt{\frac{\sigma k^{3}+g k\left(\rho_{2}-\rho_{1}\right)}{\left(\rho_{1}+\rho_{2}\right)} \operatorname{th}(k h)-\frac{k^{2} \Delta u^{2} \rho_{1} \rho_{2}}{\left(\rho_{1}+\rho_{2}\right)^{2}}}
$$

The system becomes unstable when the complex part of $\omega$ is non-zero, which provides the condition for the minimum critical velocity difference:

$$
\Delta u^{2}>\frac{\left(\rho_{1}+\rho_{2}\right)}{\rho_{1} \rho_{2}}\left(\sigma k+\frac{g\left(\rho_{2}-\rho_{1}\right)}{k}\right) \operatorname{th}(k h)
$$

The critical wavenumber is then obtained by calculating the minimum of the right-hand side of Equation (22):

$$
k_{c}=\sqrt{\frac{g\left(\rho_{2}-\rho_{1}\right)}{\sigma}}
$$

This corresponds to a theoretical value of $232 \mathrm{~m}^{-1}$. Experimentally, the measured values were equal to $k_{c}=197 \pm 58 \mathrm{~m}^{-1}$.

Moreover, if viscosity and closed-end effects are neglected, the velocity distribution at the beginning of the simulation is:

$$
\left\{\begin{array}{cl}
u_{2}=-\frac{\left(\rho_{2}-\rho_{1}\right) h_{1} g \sin (\gamma)}{\left(\rho_{1} h_{2}+\rho_{2} h_{1}\right)} t & \text { for } 0<z<h \\
u_{1}=\frac{\left(\rho_{2}-\rho_{1}\right) h_{2} g \sin (\gamma)}{\left(\rho_{1} h_{2}+\rho_{2} h_{1}\right)} t & \text { for } h<z<H
\end{array}\right.
$$

From this equation, Thorpe [1969] predicted the time of the instability onset between $1.5 \mathrm{~s}$ and $1.7 \mathrm{~s}$ and observed experimentally $\mathrm{t}_{\mathrm{onset}}=1.88 \pm 0.07 \mathrm{~s}$. Finally, he evaluated the wave velocity at $\mathrm{u}_{\mathrm{waves}}=2.38 \mathrm{~cm} / \mathrm{s}$ and observed $\mathrm{u}_{\mathrm{waves}}=2.6 \mathrm{~cm} / \mathrm{s}$. 

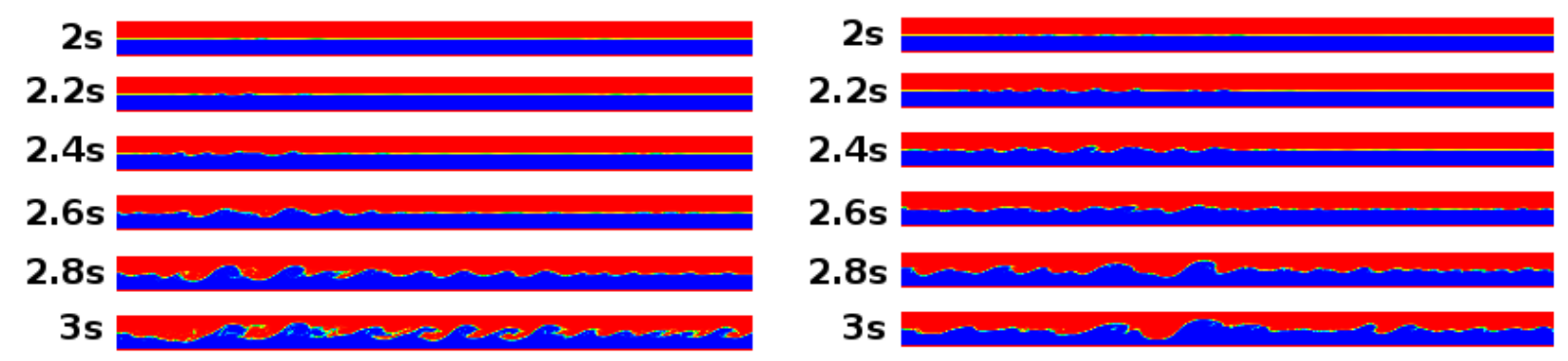

Figure 5. Influence of the drag force expression in terms of interface shape, left: previous drag force with $\tau=1.10^{-7} \mathrm{~s}$, right: new drag force given by Equation (15), only the middle 0.6 meters long section of the channel is shown.

Simulation results. Both fluid layers have the same initial height $\mathrm{h}_{1}=\mathrm{h}_{2}=1.5 \mathrm{~cm}$. The properties of the two fluids are: $\rho_{1}=780 \mathrm{~kg} / \mathrm{m}^{3}, \mu_{1}=1.5 .10^{-3} \mathrm{~Pa} . \mathrm{s}, \rho_{2}=1000 \mathrm{~kg} / \mathrm{m}^{3}$ and $\mu_{2}=1.10^{-3} \mathrm{~Pa}$.s. Surface tension is equal to $\sigma=0.04 \mathrm{~N} / \mathrm{m}$.

The dimensions of the computational domain are $\mathrm{L}=1.83 \mathrm{~m}$ and $\mathrm{H}=3 \mathrm{~cm}$ (see Figure 4). The mesh contains 80 x 4880 cells. A wall boundary condition is imposed everywhere except in front and behind, where symmetry boundary planes are defined. The simulation is performed with an adaptive time step with an initial value equal to $1 \mathrm{~ms}$ and a maximum CFL number of 0.9 .

With this test case, we observe the effect of the modification of the drag force. The results are compared in terms of interface shape, critical wavenumber, wave velocity, time of the instability onset and evolution of the maximum value of the average interface velocity at the beginning of the simulation. The following expression is used to evaluate $\mathrm{U}$ :

$$
\mathrm{U}=\frac{\varepsilon_{1}^{*} \rho_{1} u_{1}+\varepsilon_{2}^{*} \rho_{2} u_{2}}{\varepsilon_{1}^{*} \rho_{1}+\varepsilon_{2}^{*} \rho_{2}}
$$

In Figure 5, an example of the Kelvin Helmholtz instability over time is displayed for the two drag forces. A FFT analysis of the interface is performed to obtain the critical wavenumber. The results are displayed in Figure 6. The critical wavenumber obtained with the previous drag force with $\tau=1.10^{-7} \mathrm{~s}$ is equal to $174 \mathrm{~m}^{-1}$ and to $195 \mathrm{~m}^{-1}$ with the new drag force given by Equation (13). These results are in good agreement with the experimental and theoretical data. As a comparison, Bartosiewicz et al. [2008] found $143 \mathrm{~m}^{-1}$ and Štrubelj [2009] $157 \mathrm{~m}^{-1}$.

The interface is also examined in terms of amplitude growth in order to evaluate the time of the instability onset. For this purpose, the standard deviation of the interface is evaluated every $0.2 \mathrm{~s}$ until $3.2 \mathrm{~s}$. The results are presented in Figure 7. With the previous drag force with $\tau=1.10^{-7} \mathrm{~s}, \mathrm{t}_{\text {onset }}$ is found equal to $1.7 \mathrm{~s}$ and $1.6 \mathrm{~s}$ with the new drag force given by Equation (13). These results agree well with the simulations of Bartosiewicz et al. [2008] with $t_{\text {onset }}=1.9 \mathrm{~s}, \breve{S}$ Strubelj [2009] with $\mathrm{t}_{\text {onset }}=2 \mathrm{~s}$ and the theoretical and experimental data. 

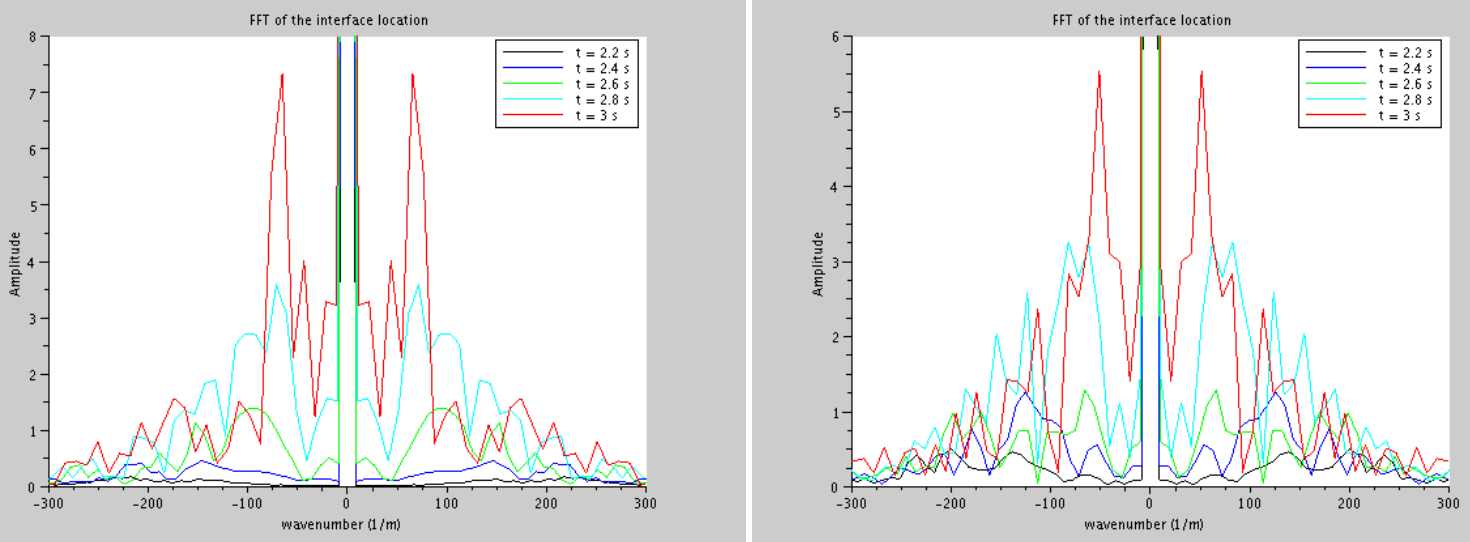

Figure 6. FFT analysis of the interface, left: previous drag force with $\tau=1.10^{-7} \mathrm{~s}$, right: new drag force given by Equation (13), only the middle 0.6 meters long section of the channel is used for this analysis.

Moreover, Figure 8 presents the maximum value of the average interface velocity $U$ over time. The new drag force is in better agreement with the theory. In fact, since the new drag force expression considers the physical properties of the flow, the numerical instabilities are reduced.

In Figure 9, the velocity profiles are displayed for the two drag forces. We can notice that, at the interface, the velocities of the two fluids are equal.

Finally, the wave speed $\mathrm{u}_{\text {waves }}$ is evaluated by calculating the crest-to-crest distance at different positions in the tube. Figure 10 gives an example of the waves used for this calculation. We find $\mathrm{u}_{\text {waves }}=3.9 \mathrm{~cm} / \mathrm{s}$ with the previous drag force with $\tau=1.10^{-7} \mathrm{~s}$ and $\mathrm{u}_{\text {waves }}=2.5 \mathrm{~cm} / \mathrm{s}$ with the new drag force given by Equation (13). These results agree well with the simulations of Bartosiewicz et al. [2008] with $u_{\text {waves }}=2.5 \mathrm{~cm} / \mathrm{s}$ and Strubelj [2009] with $u_{\text {waves }}=3 \mathrm{~cm} / \mathrm{s}$.

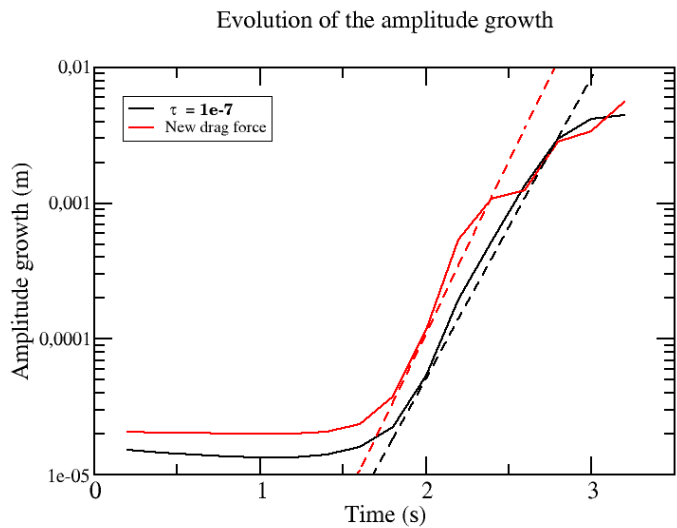

Figure 7. Amplitude growth obtained by evaluating the standard deviation of the interface over time. 


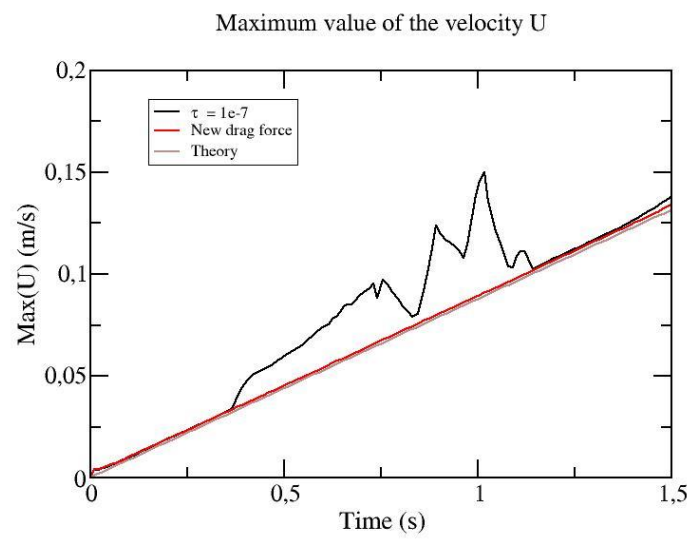

Figure 8. Average interface velocity $U$ over time, $U$ is defined in Equation (25), the theory is given by Equation (24).
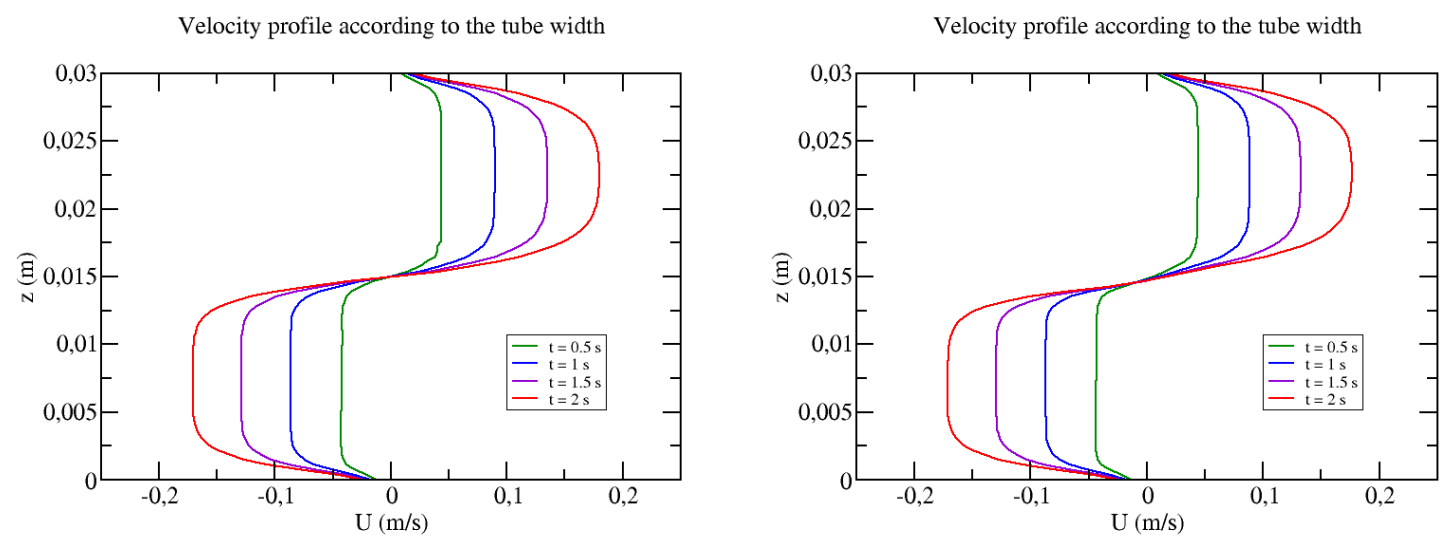

Figure 9. Variation of the average interface velocity $U$ along the tube width, left: previous drag force with $\tau=1.10^{-7} \mathrm{~s}$, right: new drag force given by Equation (13).

In Table 1, all the parameters evaluated in this simulation are summarized and compared with the theoretical and experimental data and other simulation results.
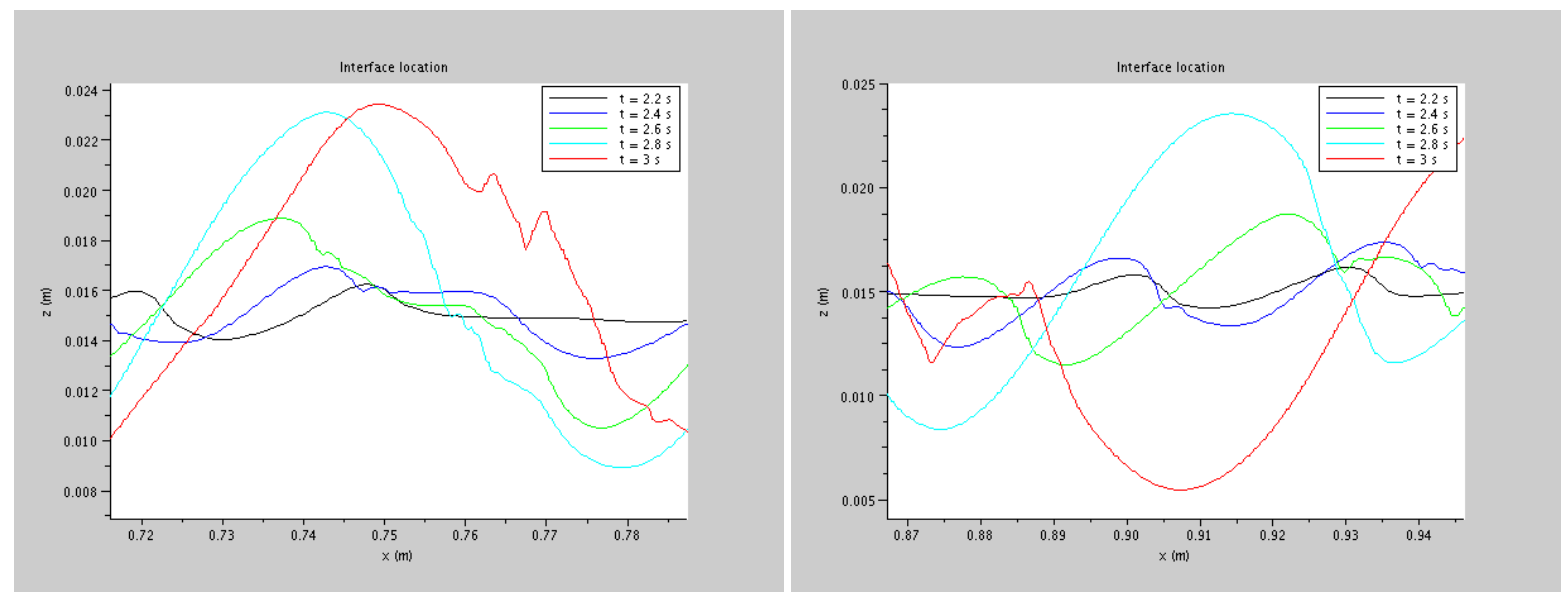

Figure 10. Physical location of the interface at different times, left: previous drag force with $\tau=1.10^{-7} \mathrm{~s}$, right: new drag force given by Equation (13). 
Table 1

Comparison between our simulation, the theoretical and experimental data and the simulations of Bartosiewicz et al. [2008] and Štrubelj [2009]

\begin{tabular}{|cccc|}
\hline Results & $k_{c}\left(\mathrm{~m}^{-1}\right)$ & $\mathrm{t}_{\text {onset }}(\mathrm{s})$ & $\mathrm{u}_{\mathrm{waves}}(\mathrm{cm} / \mathrm{s})$ \\
\hline Previous drag force, $\tau=1.10^{-7} \mathrm{~s}$ & 174 & 1.7 & 3.9 \\
\hline New drag force, Equation $(13)$ & 195 & 1.6 & 2.5 \\
\hline Theory & 232 & $1.5-1.7$ & 2.38 \\
\hline Experiments & $197 \pm 58$ & $1.88 \pm 0.07$ & 2.6 \\
\hline Bartosiewicz et al. [2008] & 143 & 1.9 & 2.5 \\
\hline Strubelj [2009] & 157 & 2 & 3 \\
\hline
\end{tabular}

Thus, we see that the flow behavior is well predicted in our simulation. Moreover, this test case highlights that the new drag force improves significantly the results.

Rayleigh-Taylor instability The Rayleigh-Taylor instability occurs in a system with two immiscible fluids of different densities in the presence of a gravity field, perpendicular to the interface. The fluid with higher density $\left(\rho_{1}=3 \mathrm{~kg} / \mathrm{m}^{3}, \mu_{1}=0.03\right.$ Pa.s) is initially located above the fluid with lower density $\left(\rho_{2}=1780 \mathrm{~kg} / \mathrm{m}^{3}, \mu_{2}=0.01\right.$ Pa.s $)$.

The aim of this test case is to compare our results to other codes.

Theory. We define the Atwood number $\mathrm{A}_{\mathrm{t}}$, which is equal to 0.5 in our case:

$$
\mathrm{A}_{\mathrm{t}}=\frac{\rho_{1}-\rho_{2}}{\rho_{1}+\rho_{2}}
$$

As long as the flow can be analyzed with linearized Navier-Stokes' equations, the amplitude of the interface deformation can be expressed as followed:

$$
\mathrm{a}(\mathrm{t})=\delta_{0} \exp (\omega t)
$$

with $\delta_{0}$ the initial amplitude of the interface equal to $1 \mathrm{~mm}$.

The pulsation $\omega$ is given by:

$$
\omega^{2}=g k \mathrm{~A}_{\mathrm{t}}+\frac{k^{2} \sigma}{\rho_{1}+\rho_{2}}
$$

with $\mathrm{g}=10 \mathrm{~m} / \mathrm{s}^{2}, k=\frac{\pi}{L}$ at first order and $\sigma=0.075 \mathrm{~N} / \mathrm{m}$.

Simulation results. The simulation is performed in a closed box $(\mathrm{H}=5 \mathrm{~m}, \mathrm{~L}=1 \mathrm{~m})$. The mesh contains $96 \times 480$ cells. A wall boundary condition is imposed at the top and the bottom of the mesh and symmetry boundary planes everywhere else. A variable time step is chosen for the simulation. Its initial value is taken equal to $1 \mathrm{~ms}$ and the maximum CFL number is fixed at 0.9 . 


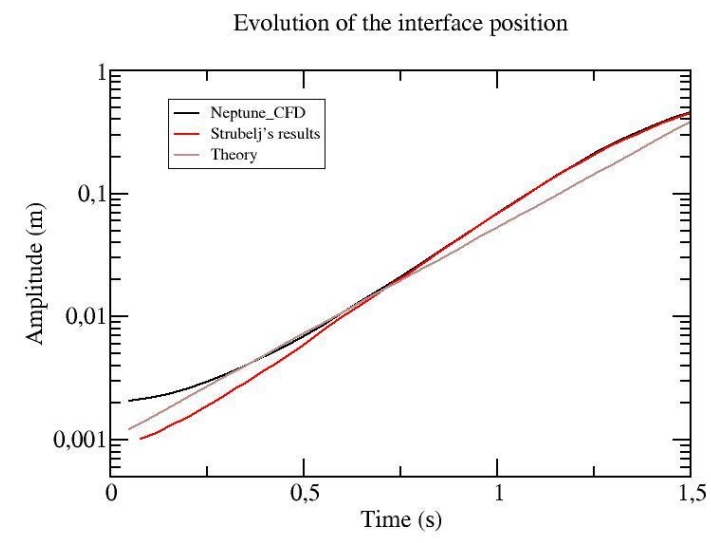

Figure 11. Interface location simulated with the code NEPTUNE_CFD using the new drag force expression compared with Štrubelj's simulation results [e.g., Strubelj 2009].

The interface between the two fluids is initialized as a small cosine wave with an amplitude equal to 1 $\mathrm{mm}$. Because the cell length is greater than $1 \mathrm{~mm}$, we use the following expression for the initialization:

$$
\delta=\delta_{0}\left(\cos \left(\frac{2 \pi \mathrm{x}}{\mathrm{L}}-\pi\right)+1\right)
$$

with $0<\mathrm{x}<\mathrm{L}$.

The simulations are performed without surface tension.

The results in terms of interface position are compared with the theory using the analytical expression (27) and the results obtained by Strubelj [2009]. Figure 11 shows that our simulation predicts well the interface position for short times. The difference observed at the very beginning is due to the interface initialization.

Figure 12 proposed a comparison of our results with other codes. We can notice that the smearing of the interface is well controlled by the use of interface sharpening or the geometrical interface reconstruction in the code FLUENT. Moreover, the shape of the mushroom obtained with the different codes remains the same except with the code FLUENT, where the mushroom is more lately extended. A major difference can be observed with the Štrubelj's in-house code [e.g., Strubelj 2009]. In fact, some particles of the above fluid are early created by the mushroom extension.

\section{CONCLUSION}

A two-fluid model coupled with a conservative level set method has been implemented in the CMFD code NEPTUNE_CFD to simulate separated flows. This approach includes an artificial compression step to control the natural interface diffusion induced by the two-fluid approach. This adding equation allows a more accurate evaluation of the local parameters such as the interface normal vector or the curvature. Moreover, because the two-fluid model can predict non zero relative velocities at large interfaces, the drag force plays an important role in the simulation of free surfaces to cancel the velocity difference between the two continuous fields. Thus, in this paper, a new expression of this force has been developed, which takes into account the physical properties of the flow. 


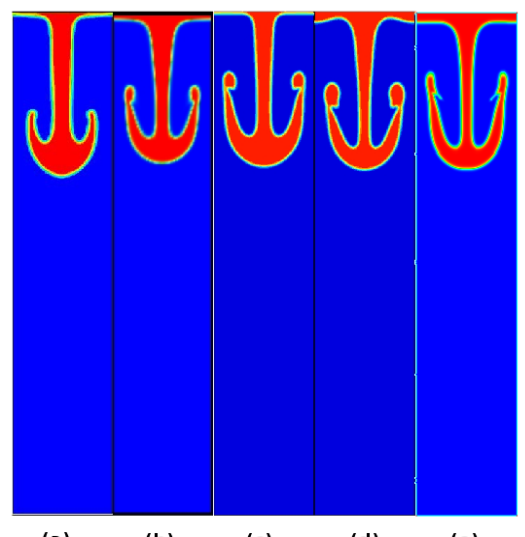

(a)

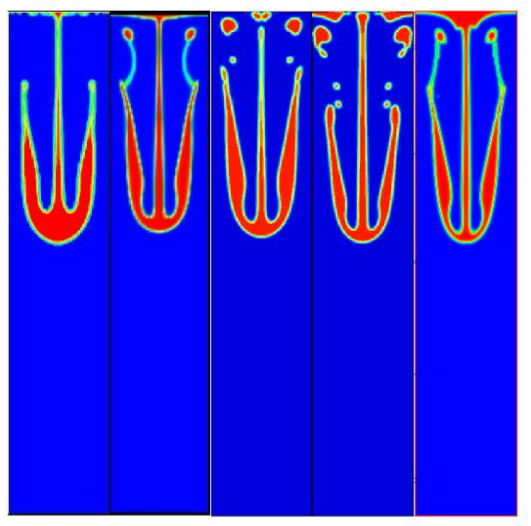

$\begin{array}{llll}\text { (a) } & \text { (b) } & \text { (c) } & \text { (d) }\end{array}$

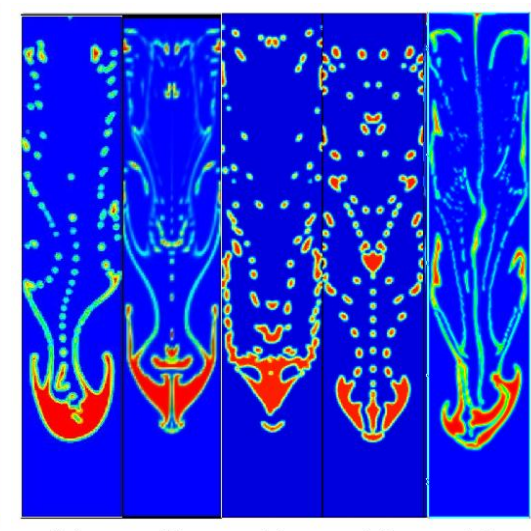

(a) (b)

(e)

Figure 12. Evolution of the Rayleigh Taylor instability obtained with various models, (a) VOF with geometrical interface reconstruction in FLUENT [e.g., Strubelj 2009], (b) VOF with interface sharpening in CFX [e.g., Strubelj 2009], (c) single-fluid model with interface sharpening with Štrubelj's in-house code [e.g., Strubelj 2009], (d) two-fluid model with interface sharpening with Štrubelj's in-house code [e.g., Strubelj 2009], (e) two-fluid model with interface sharpening in the NEPTUNE_CFD code, left to right: $\mathrm{t}=2 \mathrm{~s}, \mathrm{t}=2.5 \mathrm{~s}$ and $\mathrm{t}=3 \mathrm{~s}$.

To validate this method, a mesh refinement test has been first performed on a static bubble. The simulations showed a reasonable mesh convergence especially for the intensity of the spurious velocities, which remains low even with coarse meshes. Then, the Thorpe's experiment [e.g., Thorpe 1969] has been simulated. In this test case, the sheared interface highlights the role of the drag force to predict the flow behavior with a high level of accuracy. Our results were in good agreement with the experimental and theoretical data and the other code simulation results. Moreover, we noticed that numerical instabilities on the interface velocity prediction were produced by choosing a non physical drag force. Finally, we compared the code NEPTUNE_CFD with three other codes by simulating the Rayleigh-Taylor instability. Our results gave reasonable agreements with the other simulations.

The recent developments of the multifield approach have been included in the definition of the new drag force. Nevertheless, work is still in progress to validate the method on complex flows with two continuous fields, liquid and gas, and a gaseous dispersed field. Moreover, no turbulence models have been implemented yet with this approach. However, we can point out the work of some research groups [e.g., Larocque et al. 2010, Liovic and Lakehal 2007, Vincent et al. 2008] on the LES modeling of two-phase turbulence terms.

\section{REFERENCES}

Bartosiewicz, Y. et al. [2008], A first assessment of the NEPTUNE_CFD code: Instabilities in a stratified flow comparison between the VOF method and a two-field approach. Int. J. Heat and Fluid Flow, Vol. 29, pp 460-478.

Brackbill, J. U. et al. [1992], A continuum method for modeling surface tension, J. Comput. Phys., Vol. 100, pp 335-354.

Clift, R. et al. [1978], Bubbles, drops, and particles, Academic Press, New York.

Denèfle, R. [2014], Multifield hybrid approach for two-phase flow modeling - Part 1: Adiabatic flows, Comput. Fluids. 
Enright, D. et al. [2002], A hybrid particle level set method for improved interface capturing, $J$.

Comput. Phys., Vol. 183, pp 83-116.

Hirt, C.W., Nichols, B.D. [1981], Volume of fluid (VOF) method for the dynamics of free boundaries, J. Comput. Phys., Vol. 39, pp 201-225.

Ishii, M. [1975], Thermo-fluid dynamic, theory of two-phase flow, Eyrolles.

Lahey, R.T. and Drew D.A. [2001], The analysis of two-phase flow and heat transfer using a multidimensional, four field, two-fluid model, NED, Vol. 204, pp 29-44.

Larocque, J. et al. [2010], Parametric study of LES subgrid terms in a turbulent phase separation flow, Int. J. Heat Fluid Flow, Vol. 31, pp 536-544.

Liovic, P. and Lakehal, D. [2007], Multi-physics treatment in the vicinity of arbitrarily deformable gas liquid interfaces, J. Comput. Phys., Vol. 222, pp 504-535.

Mimouni, S. et al. [2011], Combined evaluation of second order turbulence model and polydispersion model for two-phase boiling flow and application to fuel assembly analysis, $N E D$, Vol. 241, Issue 11, pp 4523-4536.

Mimouni, S. et al. [2014], Multifield approach and interface locating method for two-phase flows in nuclear power plant, submitted to $N E D$.

Olsson, E., and Kreiss, G. [2005], A conservative level set method for two phase flow, J. Comput. Phys., Vol. 210, pp. 225-246.

Patankar, S. and Spalding, D. [1972], A calculation procedure for heat, mass and momentum transfer in three-dimensional parabolic flows, Int. J. Heat Mass Transf., Vol. 15, No. 10, pp 1787-1806.

Raymond, F., and Rosant, J.-M. [2000], A numerical and experimental study of the terminal velocity and shape of bubbles in viscous liquids, Chem. Eng. Sc., Vol. 55, pp 943-955.

Schiller, L., and Nauman, A. [1935], A drag coefficient correlation, V.I.D. Zeitung, Vol. 77, pp 318320.

Štrubelj, L. [2009], Numerical simulations of stratified two-phase flows with two-fluid model and interface sharpening, M.Sc. Thesis, University of Ljublana.

Sussman, M. et al. [1994], A level set approach for computing solutions to incompressible two-phase flow, J. Comput. Phys., Vol. 114, pp 146-159.

Thorpe, S. [1969], Experiments on the instability of stratified shear flows: immiscible fluids, $J$ Fluid Mech., Vol. 39, pp 25-48.

Unverdi, S.O. and Tryggvason, G. [1992], A front-tracking method for viscous, incompressible, multifluid flows, J. Comput. Phys., Vol. 10, pp 25-37.

Vincent, S. [2008], Numerical simulation of phase separation and a priori two-phase LES filtering, Comput. Fluids, Vol. 37, pp 898-906. 
Zuzio, D. et al. [2013], Numerical simulation of primary and secondary atomization, C. R. Mecanique, Vol. 241, pp 15-25. 